\section{Cutaneous melanomas in rabbits: rare but often fatal}

\author{
Martin Hammer, ${ }^{1}$ Frank Weigner, ${ }^{2}$ \\ Robert Klopfleisch2
}

1Small Animal Clinic Rostock, Germany;

2Freie Universität Berlin, Institute of

Veterinary Pathology, Berlin, Germany

\section{Abstract}

An adult male dwarf rabbit (Oryctolagus cuniculus) was presented to the veterinarian due to hind limb lameness. The rabbit was in a reduced body condition. Clinical examination and cytology identified a cutaneous melanoma in the inguinal region. Whole body radiographs identified multifocal radio-opaque masses in both lungs which where assumed to be lung metastases. The animal was euthanized due to the poor prognosis. Necropsy confirmed a malignant, melanotic melanoma with pulmonary and hepatic metastases. Histopathologically, the primary tumor and the metastases were composed of epitheloid cells which showed infiltrative growth. The rabbit was diagnosed with metastatic, cutaneous, melanotic melanoma. Melanomas in rabbits can be recognized as highly malignant independent on their pigmentation status. Pulmonary tropism seems to be a distinct feature of this tumor type in rabbits and indicates that a comprehensive diagnostic workup is necessary to avoid anesthesia-related incidents.

\section{Introduction}

Neoplastic diseases are of minor relevance in rabbits kept as farm animals when compared to infectious diseases. However, with the increasing popularity of rabbits as pets and the associated increased life span, an increased incidence of neoplastic diseases can be observed in veterinary practice and pathology departments. The most common tumors in rabbits are uterine carcinomas, thymomas, lymphomas but neoplasias of almost all other organs and tissues have been described in single case reports. ${ }^{1-4}$ Melanomas and particularly malignant melanomas are certainly not the most common tumors of the rabbit but regularly appear in the veterinary practice.1,2,5 The present case report exemplifies the typical course of the disease of a rabbit with a malignant cutaneous melanoma with metastatic spread to the lung and the liver and reviews the recent literature on melanomas in rabbits.

\section{Case Report}

A male, 4-year-old, $1.5 \mathrm{~kg}$ dwarf rabbit (Oryctolagus cuniculus) was presented to the referring veterinarian due to lameness of the hind limbs. The animal lived outdoors and the exact time point of the onset of the lameness and a potential trauma was not reported by the owner. In addition, the animal never received a vaccination and was not dewormed.

\section{Clinical findings}

The animal had a mildly reduced general body condition (body condition score 4/9) with a feculent ano-genital region. Rectal temperature was $38^{\circ} \mathrm{C}\left(100.4^{\circ} \mathrm{F}\right)$ and oral cavity and teeth were unsuspicious and the oral mucosa pinkish. Auscultation found a heart rate of 250 beats/min (reference range: 130 to 325 beats/min) and a respiratory rate of 50 breaths/min (reference range, 32 to 60 breaths/min). Auscultation of the lung and the abdomen found no abnormalities. Front and hind limbs were in a physiologic position, no crepitation was found and all areas showed a normal superficial sensitivity. In the inguinal region close to the perianal glands there was a solid, indolent, $4 \times 4 \times 1 \mathrm{~cm}$ in diameter large, black mass with multifocal epidermal ulceration (Figure 1). A fine needle aspirate of the mass was stained with Diff-Quick and identified aggregated and single epithelial cells with melanin-containing granules. This led to the diagnosis of a melanotic cutaneous melanoma. Latero-lateral (LL) and ventro-dorsal (VD) whole body radiographs were taken to evaluate a potential metastatic spread of the tumor and to estimate the potential success of a surgical excision of the tumor. Radiographs were taken in VD and LL position without anesthesia (42 $\mathrm{kV}, 10 \mathrm{mAs}$ ). Within in the lung there were multifocally distributed, radiopaque masses, which were considered as pulmonary metastases of the cutaneous melanoma (Figure 2 A,B). Due to the poor prognosis and the limited operability the animal was euthanized and submitted for full body necropsy.

\section{Pathological findings}

At necropsy, the animal was in good nutritional condition. The diagnosis of a cutaneous, melanotic melanoma mass of the inguinal region was confirmed. The primary tumor was not encapsulated, showed and infiltrative growth and had a mottled whit and black surface on cut section. Multifocal, heavily pigmented, up to $1 \mathrm{~cm}$ in diameter large pleural (Figure 3) and pulmonary (Figure 4) were identified in the thorax. In addition, partly pigmented metastases were also identified in the liver. Distant metastases were not observed in the regional lymph node and other organs. Finally, the heart showed a mild hypertrophy of
Correspondence: Dr. Robert Klopfleisch, Freie Universität Berlin, Institute of Veterinary Pathology, Robert-von-Ostertag-Str. 15, 14163 Berlin, Germany.

Tel. +49.30.83862450 - Fax: +49.30 .83862522 .

E-mail: klopfleisch.robert@vetmed.fu-berlin.de

Key words: rabbit, melanoma, metastasis, skin, lung, liver.

Acknoledgments: the preparation of the manuscript and the related diagnostic work has been funded intramuraly by the Institute of Veterinary Pathology and the Small animal clinic Rostock.

Received for publication: 1 September 2011. Accepted for publication: 26 September 2011.

This work is licensed under a Creative Commons Attribution 3.0 License (by-nc 3.0).

CC Copyright M. Hammer et al., 2010 Licensee PAGEPress, Italy

Veterinary Science Development 2011; 1:e9 doi:10.4081/vsd.2011.e9

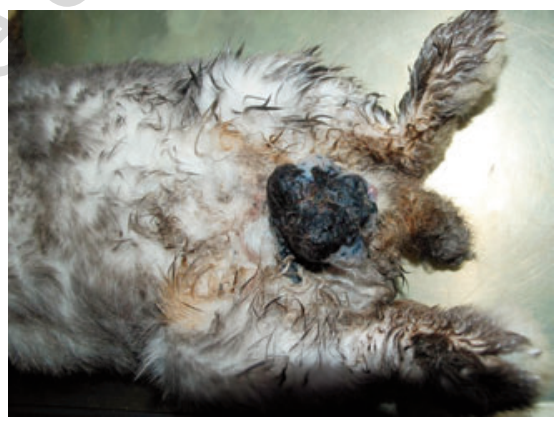

Figure 1. Cutaneous, malignant melanoma in the inguinal region, rabbit. The black, partly ulcerated, indolent mass had a maximum size of $4 \times 4 \times 1 \mathrm{~cm}$ in diameter.
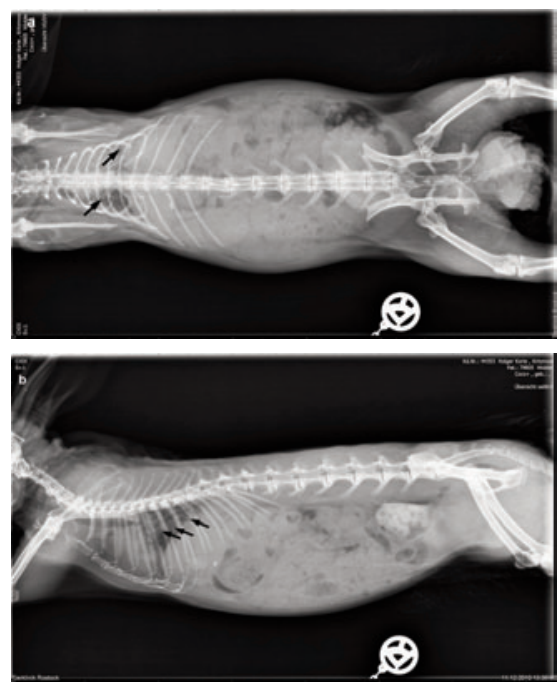

Figure 2. Latero-lateral (A) and dorso-ventral (B) radiograph, rabbit. Multifocal, well circumscribed shadows (arrows) were visible in both lung lobes, which were identified as metastases of the melanoma at necropsy. 
the right atrium and ventricle, most probably as a consequence of the lung metastases.

Histopathologically, the diagnosis of a malignant cutaneous melanoma with thoracic and hepatic metastases was confirmed. Tumor cells of both primary tumor and metastases were epitheloid in shape, had anisokaryosis with large nucleoli and an erratic pigmentation (Figure 5).

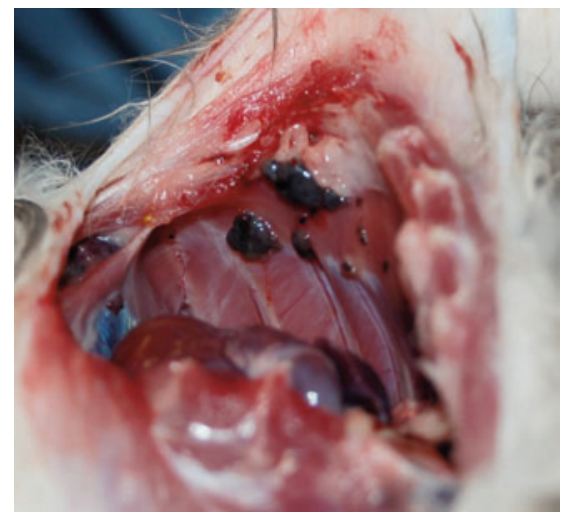

Figure 3. Pleural metastases of a malignant melanoma, rabbit. Multiple, up to $1 \mathrm{~cm}$ in diameter large, black, metastases of a cutaneous melanoma were present on the pleural surface. There were no signs of an imprinting of the metastases from the lung.

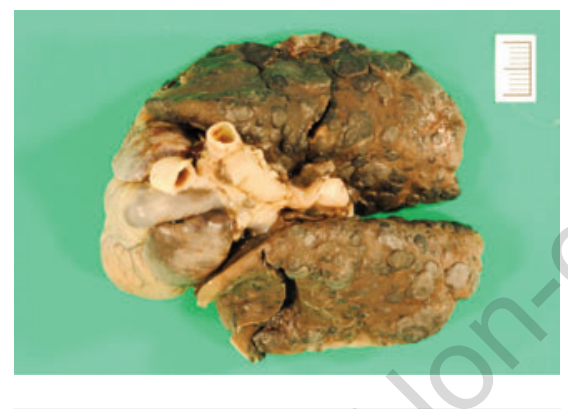

Figure 4. Pulmonary metastases of a cutaneous melanoma, formalin-fixed lung, rabbit. Up to $40 \%$ of the lung parenchyma was replaced by metastases of a cutaneous melanoma.

\section{Discussion}

The clinical and pathologic diagnosis of melanomas by cytological and histopathologic examination is relatively simple due to the high percentage of melanotic tumors. ${ }^{1,2}$ In contrast, amelanotic melanomas are rare in the rabbit but may complicate the diagnosis and occasionally require immunohistologic confirmation of the diagnosis by the detection of melanocyte markers like Melan-A.1,2 A breed, sex or age predisposition has not been reported for rabbits but older animals and male animals seem to be overrepresented.1,2,5 There are variable reports on the malignancy of cutaneous melanomas in the rabbit but in the majority of reported cases the primary tumors had an infiltrative growth with invasion of lymph and blood vessels in the tumor vicinity.1,2,5 Taken all the reports together the incidence of distant metastases seems to be around $50 \%$, similar to that of cutaneous melanomas on other species such as horses, pigs and dogs.1,2,5-7 The present case exemplifies the highly aggressive behavior and the high metastatic potential of cutaneous, malignant melanomas in rabbits. The tropism of the metastatic tumor cells towards thoracic structures like the pleura and the lung seems to be

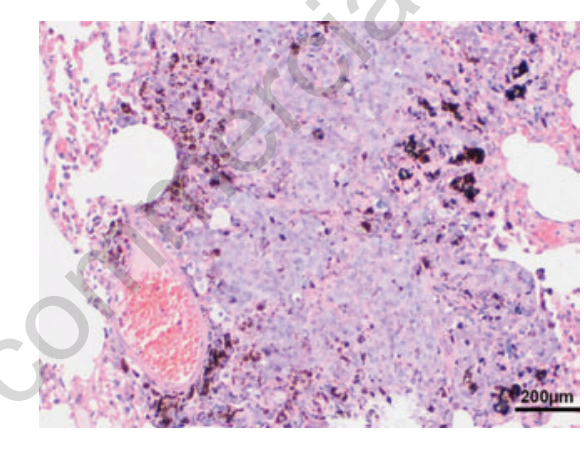

Figure 5. Pulmonary metastases of a cutaneous melanoma, lung, rabbit. Metastatic melanoma cells were identified in all areas of the lung. The partly melanin-containing tumor cells showed a perivascular, invasive growth into the surrounding lung tissue. (H\&E-staining). Scale bar $=200 \mu \mathrm{m}$. a distinctive feature of rabbit melanomas and has been described in other reports before. ${ }^{2,5}$ Furthermore, the present case shows that the high risk of lung metastases of these tumors requires a comprehensive clinical and radiographic examination to evaluate the clinical prognosis and the anesthetic risk of rabbits affected by this tumor type.

In conclusion, the present case of a metastatic cutaneous melanoma in a dwarf rabbit shows that cutaneous melanomas although less often than uterine adenocarcinomas and lymphoma are often fatal and require a comprehensive pre-surgical clinical diagnostic examination to evaluate further treatment options and to avoid anesthesia-related incidents.

\section{References}

1. von Bomhard W, Goldschmidt MH, Shofer FS, et al. Cutaneous neoplasms in pet rabbits: a retrospective study. Vet Pathol 2007;44:579-88.

2. Zerfas PM, Brinster LR, Starost MF, et al. Amelanotic melanoma in a New Zealand White Rabbit (Oryctolagus cuniculus). Vet Pathol 2010;47:977-81.

3. Rusk GY, Epstein N. Adenocarcinoma of the Uterus in a Rabbit. Am J Pathol 1927; 3:235-40 7 .

4. Vernau KM, Grahn BH, Clarke-Scott HA, Sullivan N. Thymoma in a geriatric rabbit with hypercalcemia and periodic exophthalmos. J Am Vet Med Assoc 1995;206: 820-2.

5. Hotchkiss CE, Norden H, Collins BR, Ginn PE. Malignant melanoma in two rabbits. Lab Anim Sci 1994;44:377-9.

6. Millikan LE, Boylon JL, Hook RR, Manning PJ. Melanoma in Sinclair swine: a new animal model. J Invest Dermatol 1974;62: 20-30.

7. Valentine BA. Equine melanocytic tumors: a retrospective study of 53 horses (1988 to 1991). J Vet Intern Med 1995;9:291-7. 